\title{
Fetal growth and psychiatric and socioeconomic problems: population-based sibling comparison
}

\author{
Quetzal A. Class, Martin E. Rickert, Henrik Larsson, Paul Lichtenstein and Brian M. D'Onofrio
}

\section{Background}

It is unclear whether associations between fetal growth and psychiatric and socioeconomic problems are consistent with causal mechanisms.

\section{Aims}

To estimate the extent to which associations are a result of unmeasured confounding factors using a sibling-comparison approach.

\section{Method}

We predicted outcomes from continuously measured birth weight in a Swedish population cohort $(n=3291773)$, while controlling for measured and unmeasured confounding.

\section{Results}

In the population, lower birth weight $(\leqslant 2500 \mathrm{~g})$ increased the risk of all outcomes. Sibling-comparison models indicated that lower birth weight independently predicted increased risk for autism spectrum disorder (hazard ratio for low birth weight $=2.44,95 \% \mathrm{Cl}$ 1.99-2.97) and attention-deficit hyperactivity disorder. Although attenuated, associations remained for psychotic or bipolar disorder and educational problems. Associations with suicide attempt, substance use problems and social welfare receipt, however, were fully attenuated in sibling comparisons.

\section{Conclusions}

Results suggest that fetal growth, and factors that influence it, contribute to psychiatric and socioeconomic problems.

\section{Declaration of interest}

None.
Indicators of poor fetal growth, such as low birth weight $(\leqslant 2500 \mathrm{~g})$, are linked with increased offspring risk for neurodevelopmental disorders, ${ }^{1-4}$ academic problems ${ }^{5-8}$ and poor social outcomes. ${ }^{5}$ The developmental origins of health and disease hypothesis, which states that developmental plasticity contributes to fetal physiological adaptations made in response to the intrauterine environment, has been used to explain the identified associations. ${ }^{9}$ The mechanisms linking fetal growth with later psychiatric, academic and social problems are not straightforward, however. Impaired fetal growth may act as an independent, environmental risk factor, as twin studies have shown for attention-deficit hyperactivity disorder (ADHD). ${ }^{10-12}$ Previously identified associations may also be a result of unmeasured selection factors, such as environmental confounding or shared genetic liability, that influence both the likelihood of experiencing the risk and the outcome. ${ }^{13,14}$ For example, low birth weight is associated with environmental risks that are themselves predictive of subsequent adverse outcomes ${ }^{15}$ and family and twin studies indicate that genetic and shared environmental factors influence birth weight. ${ }^{16}$ As such, the field should remain cautious in drawing causal conclusions between fetal growth and these outcomes. Conflicting results across outcomes also have been found, ${ }^{1,5,17-20}$ and a recent meta-analysis showed that associations between low birth weight and depression may be as the result of publication bias. ${ }^{19}$ Further, previous studies have been limited by self- and parent-report of both risk and outcome. ${ }^{1,21}$ Thus, analyses that determine precise and accurate estimates of the strength of the associations, as well as those that begin to pull apart genetic and environmental influences, are needed in the field. ${ }^{19,22}$

We sought to rigorously examine the associations between fetal growth and psychiatric and socioeconomic problems in a Swedish population cohort. Our outcomes included autism spectrum disorder (ASD), ADHD, psychotic or bipolar disorder, substance use problems, suicide attempts, criminality, failing grades in grade 9 (13-16 years old), low educational attainment and social welfare receipt. We estimated the magnitude of the associations from traditional, population-based epidemiological designs and examined the effect sizes using sibling-comparison models. Quasi-experimental approaches, such as sibling-comparison approaches, utilise design features to test alternative explanations. ${ }^{23}$ By accounting for genetic and environmental factors that make siblings similar, sibling comparisons offer a way to pull apart genetic and environmental confounding. ${ }^{23,24}$ Sensitivity analyses were used to test alternative explanations and address limitations inherent in the sibling-comparison approach. ${ }^{25}$

\section{Method}

\section{Sample}

After approval from the Institutional Review Boards at Karolinska Institutet and Indiana University, we created a prospective national cohort by linking information in the following Swedish registries: (a) the Medical Birth Register includes data on more than $99 \%$ of all pregnancies in Sweden; (b) the Multi-Generation Register contains information about the biological relationships for all individuals living in Sweden; (c) the Migration Register contains information on dates of migration in or out of Sweden; (d) the Cause of Death Register supplies dates and causes of all deaths; (e) the Patient Register contains diagnoses for all in-patient hospital admissions since 1973 and out-patient care since 2001; (f) the National Crime Register includes information about all criminal convictions; (g) the National School Register includes all subject grades at the end of grade 9 since 1983; (h) the Education Register contains information on the highest level of completed formal education; and (i) the longitudinal integration database for health insurance and social studies (LISA) contains yearly assessments of childbearing, marital and social welfare status for all individuals at least 15 years old since 1990. Detailed information about these registers is available in the online data supplement and elsewhere. ${ }^{26}$

The data-set began with 3619712 offspring born from 1973 to 2008. We removed multiple births $(86273,2.4 \%)$ because birth outcomes are different in multiples as compared with singleton births. ${ }^{27}$ We also removed offspring with missing birth weight 
information $(9888,0.3 \%)$ as well as recorded gestational age values of less than 23 weeks or greater than 42 weeks and 6 days (49374, 1.4\%). Offspring with no information regarding gender $(3,<0.1 \%)$, invalid parity information $(20,<0.1 \%)$ and those who had emigrated within the 25 year period $(182223,5.0 \%)$ were removed. We also excluded offspring missing maternal identification numbers $(158,<0.1 \%)$. The final sample consisted of 3291773 offspring born to 1735250 distinct biological mothers, representing $90.9 \%$ of all recorded Swedish births within the year range investigated.

Both ASD and ADHD were identified using in-patient and out-patient assessment information ${ }^{28}$ from individuals born between 1980 and $2001(n=2032803)$. In addition, we used a 2 -year age criterion for ASD and ADHD diagnosis. For criminality, we used an age criterion of 15 years because of the Swedish legal age of responsibility. Therefore, the criminality subsample spanned the years 1973-1994 and included 2044992 individuals. For all other outcomes, we used a 12 year age criterion. Therefore, this subsample included 2308032 offspring born between 1973 and 1997.

\section{Measures}

Birth weight

To assess fetal growth, we utilised two different representations of birth weight while controlling for gestational age at birth. For the ordinal representation, birth weight was grouped into the following ranges: $\leqslant 2500 \mathrm{~g}, 2501-3000 \mathrm{~g}, 3001-3500 \mathrm{~g}, 3501-4000 \mathrm{~g}$ (referent) and $\geqslant 4001 \mathrm{~g}$. Continuously measured birth weight was converted to a linear scale centred at $3750 \mathrm{~g}$ (reference 0 point), the approximate mean of the sample.

\section{Offspring outcomes}

We predicted six indices of psychiatric problems previously shown to be reliable measures. ${ }^{28-32}$ In particular (a) ASD and (b) ADHD were indexed using validated ${ }^{28,33}$ in-patient and out-patient diagnoses according to ICD- $9^{34}$ and ICD- $10^{35}$ for offspring born between 1980 and 2001 and being at least 2 years old at the time of diagnosis. As the ICD follows a strict definition of ADHD and ASD, results apply to the most severe cases of these disorders. In addition, it was not possible to classify ADHD according to subtype (i.e. combined, primarily hyperactive-impulsive and primarily inattentive type), since these were not recorded across the registers using the ICD. Offspring had to have been at least 12 years old to receive any of the following disorders: (c) psychotic or bipolar disorder was defined as first in-patient admission for schizophrenia, bipolar disorder or another non-organic psychotic disorder according to ICD- $8,{ }^{36}-9$ and -10 criteria $^{29}$ (d) substance use problems was defined as first in-patient admission for a primary or secondary diagnosis of alcohol or any other non-nicotine substance use disorder; ${ }^{30}$ (e) age at suicide attempt was gathered using in-patient admission for a primary or secondary diagnosis; ${ }^{31}$ and (f) criminality was indicated by the first occurrence of any criminal conviction from age 15 years, the age of legal responsibility in Sweden. ${ }^{32}$ We chose not to examine broadly defined affective disorder because in-patient admissions for that diagnosis may indicate the presence of co-occurring suicidality or psychosis, and we had access to validated indicators of these associated possible outcomes. ${ }^{29,31}$ Respective ICD codes are presented in online Table DS1, and it should be noted that clinical evaluations, not structured interviews, were used by diagnosing medical providers to determine primary diagnoses.

We predicted three indices of socioeconomic outcomes: (a) failing grades indexed poor school performance in grade 9 commensurate with a mean failing grade across 16 academic subjects; ${ }^{37}$ (b) education under 10 years was an indication of low educational attainment ${ }^{38}$ and (c) social welfare receipt, which was defined as the age of first receipt of government social welfare subsidies. For verification and converging support of these outcomes, we also predicted low income and higher education (further explained in online Fig. DS2).

\section{Covariates}

The Medical Birth Register provided offspring gender, birth order, year of birth and gestational age at birth. Measured maternal and paternal covariates included age at the offspring's birth, highest level of completed education by 2008 (to capture some socioeconomic variability across families) and lifetime history of any criminal conviction. All covariates were associated with both birth weight and the outcomes.

\section{Analyses}

We used Cox survival analysis for right-censored outcomes because not all offspring have lived through the study period. If offspring did not receive a diagnosis within the study period, they contributed person-time at risk until death, emigration or the end date of follow-up (31 December 2009), whichever came first. We used logistic regression analyses when predicting failing grades and education under 10 years because they were dichotomous outcomes. Thus, results are presented as hazard ratios (HRs) or odds ratios (ORs).

We fit a series of models for each outcome. All models controlled for offspring gender, birth order and measures of linear and quadratic gestational age. Logistic models also controlled for offspring year of birth. Because we always adjusted gestational age at birth, our predictor may be considered an index of fetal growth. The first statistical model used the ordinal representation of birth weight to estimate clinically interpretable estimates of risk across outcomes. Second, we used a continuous representation of birth weight in two baseline models. One baseline model included both a linear and quadratic representation of birth weight, whereas the other baseline model only included the linear representation of birth weight. Akaike information criterion, a measure of relative merit that penalises for model complexity, was used to select the best fitting model, either linear or quadratic. Third, we included offspring-specific (gender, birth order, linear and quadratic gestational age and maternal and paternal age at childbearing) and parental-specific covariates (maternal and paternal highest level of education and history of criminal conviction) in an adjusted model of either continuous linear or quadratic representation of birth weight. Fourth, we fitted a fixed-effects model that clustered at the maternal level, which accounted for factors that siblings share, including all genetic and environmental factors that make siblings similar. ${ }^{24}$ Covariates that may vary between siblings (i.e. offspring gender, birth order, gestational age and offspring year of birth (in logistic models)) were included in fixed-effects models. Siblings were identified as individuals sharing a biological mother (for example full or maternal half-siblings).

\section{Sensitivity analyses}

We ran several sensitivity analyses to test for biases because of preterm births, to examine whether there was converging evidence across related socioeconomic outcomes and to check assumptions inherent in the sibling-comparison design.

\section{Results}

Table 1 presents cohort demographics by birth weight category. Table 2 presents the number of offspring across outcomes by birth weight category. 


\begin{tabular}{|c|c|c|c|c|c|c|c|c|c|c|}
\hline \multirow[b]{2}{*}{ Covariates } & \multicolumn{10}{|c|}{ Birth weight category, g } \\
\hline & \multicolumn{2}{|c|}{$\begin{array}{c}\leqslant 2500 \\
(n=114580)\end{array}$} & \multicolumn{2}{|c|}{$\begin{array}{c}2501-3000 \\
(n=366500)\end{array}$} & \multicolumn{2}{|c|}{$\begin{array}{c}3001-3500 \\
(n=1075447)\end{array}$} & \multicolumn{2}{|c|}{$\begin{array}{c}3501-4000 \\
(n=1152337)\end{array}$} & \multicolumn{2}{|c|}{$\begin{array}{c}\geqslant 4000 \\
(n=583909)\end{array}$} \\
\hline \multicolumn{11}{|c|}{ Offspring, birth year: 1973-2008 $(n=3291773)$} \\
\hline Female, $n(\%)^{a}$ & 58657 & 51.19 & 207481 & 56.61 & 573332 & 53.31 & 533202 & 46.27 & 220119 & 37.70 \\
\hline Gestational age, days: mean (s.d.) & 245.46 & 25.53 & 271.19 & 12.10 & 278.14 & 9.41 & 282.14 & 8.27 & 285.24 & 7.77 \\
\hline \multicolumn{11}{|c|}{ Maternal, birth year: 1924-1995 $(n=1732107)$} \\
\hline Age at birth, years: mean (s.d.) & 28.85 & 5.67 & 28.43 & 5.36 & 28.59 & 5.19 & 28.96 & 5.10 & 29.55 & 5.06 \\
\hline Nationality, Swedish: $n$ (\%) & 54045 & 83.77 & 169706 & 83.00 & 491255 & 84.77 & 518302 & 87.12 & 254761 & 88.68 \\
\hline Upper secondary education, ${ }^{\mathrm{b}}$ n (\%) & 31870 & 49.35 & 105639 & 51.62 & 323120 & 55.71 & 347214 & 58.32 & 170492 & 59.31 \\
\hline Adult severe psychopathology, $n$ (\%) & 1748 & 2.71 & 4493 & 2.20 & 10606 & 1.83 & 9629 & 1.62 & 4377 & 1.52 \\
\hline Criminality, $n(\%)$ & 8939 & 13.84 & 26443 & 12.92 & 65981 & 11.38 & 61356 & 10.31 & 28207 & 9.81 \\
\hline \multicolumn{11}{|c|}{ Paternal, birth year: 1904-1993 $(n=1725359)$} \\
\hline Age at birth, years: mean (s.d.) & 31.75 & 6.55 & 31.38 & 6.28 & 31.49 & 6.07 & 31.78 & 5.96 & 32.28 & 5.91 \\
\hline Nationality, Swedish: $n$ (\%) & 52049 & 83.61 & 165781 & 82.69 & 485000 & 84.50 & 518434 & 87.04 & 259048 & 89.02 \\
\hline Upper secondary education, ${ }^{\mathrm{b}} \mathrm{n}(\%)$ & 27192 & 43.63 & 90199 & 44.92 & 274812 & 47.82 & 294778 & 49.44 & 145192 & 49.84 \\
\hline Adult severe psychopathology, $n$ (\%) & 1345 & 2.16 & 4033 & 2.01 & 10059 & 1.75 & 9399 & 1.58 & 4172 & 1.43 \\
\hline Criminality, $n(\%)$ & 25953 & 41.64 & 81223 & 40.45 & 219628 & 38.22 & 216353 & 36.28 & 101834 & 34.96 \\
\hline
\end{tabular}

a. Percentage of individuals by birth weight group, for offspring, the total number by birth weight group is listed in the column header, for mother and father variables, the total number of distinct mothers and fathers are listed in the left column and percentages are based on the number of non-missing cases for each variable.

b. Upper secondary education: minimum 3 years.

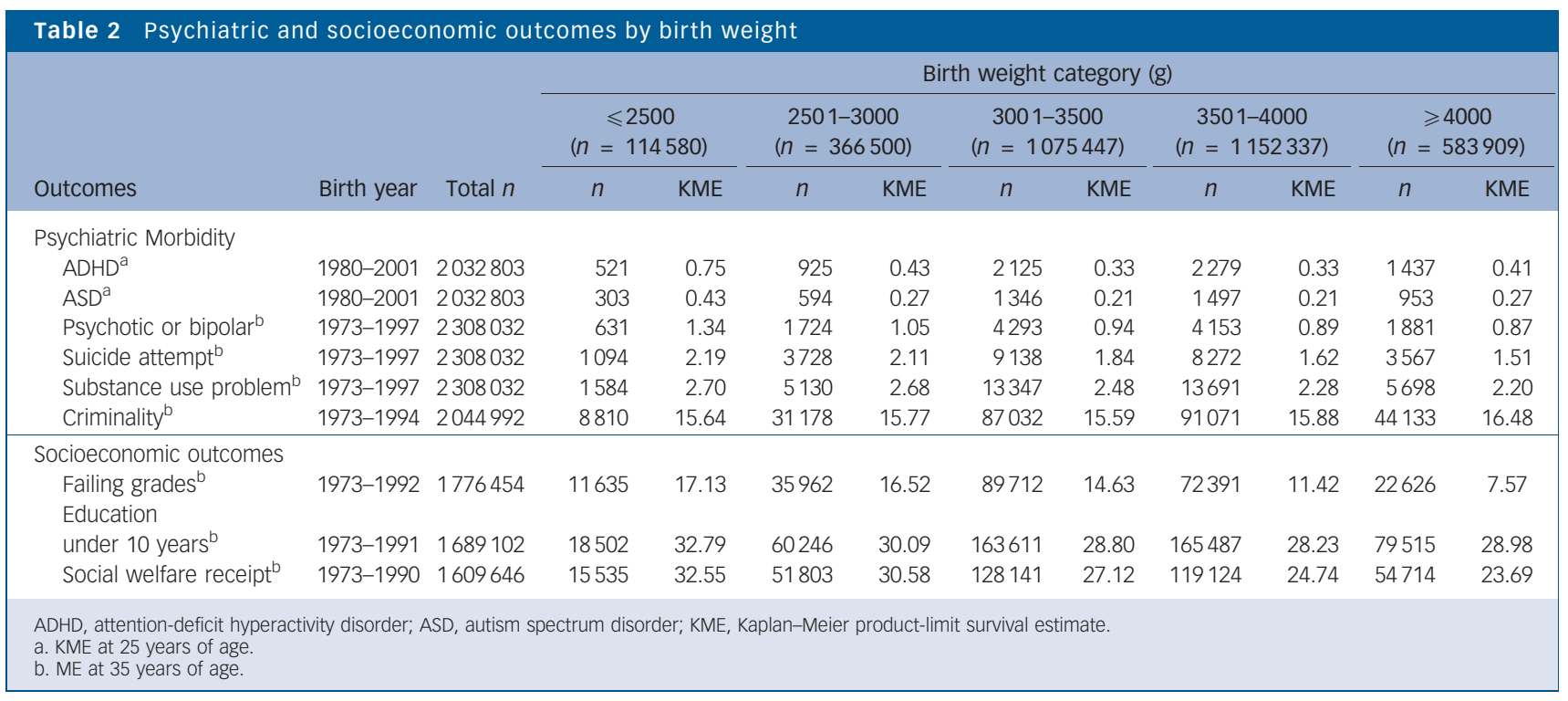

\section{Psychiatric problems}

Figure 1 presents results from the baseline ordinal model (dark bars) with $95 \%$ confidence intervals. The corresponding results using the continuous measure of birth weight (the solid line in Fig. 1) also illustrate how fetal growth was associated with later psychiatric problems. For ease of interpretation, ordinal results are discussed here and continuous results are presented graphically.

Figure $1(\mathrm{a})-(\mathrm{c})$ present the strong inverse association between birth weight and ASD $\left(\mathrm{HR}_{\text {Birth weight }(\mathrm{BW}): \leqslant 2500 \mathrm{~g}}=1.79,95 \% \mathrm{CI}\right.$ 1.64-1.96), ADHD ( $\left.\mathrm{HR}_{\mathrm{BW}: \leqslant 2500 \mathrm{~g}}=1.54,95 \% \mathrm{CI} 1.44-1.65\right)$, and psychotic or bipolar disorder $\left(\mathrm{HR}_{\mathrm{BW}: \leqslant 2500 \mathrm{~g}}=1.19,95 \% \mathrm{CI}\right.$ 1.09-1.29) respectively. The associations remained robust when adjusting for offspring- and parental-specific covariates (not shown; see online Table DS2). Also in Fig. 1, the findings from fixed-effects modelling, which compared differentially exposed siblings (light bars with 95\% confidence intervals and the dotted line), showed consistently elevated effect sizes for these outcomes. Fetal growth was associated with ASD $\left(\mathrm{HR}_{\mathrm{BW}: \leqslant 2500 \mathrm{~g}}=2.44,95 \%\right.$
CI 1.99-2.97), $\mathrm{ADHD}\left(\mathrm{HR}_{\mathrm{BW}: \leqslant 2500 \mathrm{~g}}=1.65,95 \%\right.$ CI $\left.1.40-1.93\right)$ and psychotic or bipolar disorder $\left(\mathrm{HR}_{\mathrm{BW}: \leqslant 2500 \mathrm{~g}}=1.24,95 \% \mathrm{CI}\right.$ 1.02-1.51) independent of the measured covariates and the comparison of differentially exposed siblings, consistent with a causal inference.

A different pattern of results was found when predicting suicide attempt and substance use problems (Fig. 1(d) and (e)), however. As can be noted in the dark bars in Fig. 1, population models suggested that lower birth weight increased the risk for suicide attempt $\left(\mathrm{HR}_{\mathrm{BW}: \leqslant 2500 \mathrm{~g}}=1.19,95 \% \mathrm{CI} 1.11-1.28\right)$ and substance use problems $\left(\mathrm{HR}_{\mathrm{BW}: \leqslant 2500 \mathrm{~g}}=1.27,95 \%\right.$ CI $\left.1.20-1.34\right)$. After adjusting for measured covariates (not shown; see online Table DS2) and in fixed-effects models (Fig. 1, light bars), the associations with suicide attempt $\left(\mathrm{HR}_{\mathrm{BW}: \leqslant 2500 \mathrm{~g}}=0.94,95 \% \mathrm{CI}\right.$ $0.81-1.10)$ and substance use problems $\left(\mathrm{HR}_{\mathrm{BW}: \leqslant 2500 \mathrm{~g}}=0.93\right.$, 95\% CI $0.83-1.04$ ) were fully attenuated.

The pattern of association was distinct when predicting criminality (Fig. 1(f)). More specifically, while population models showed that lower birth weight increased the risk for criminality 
(a)
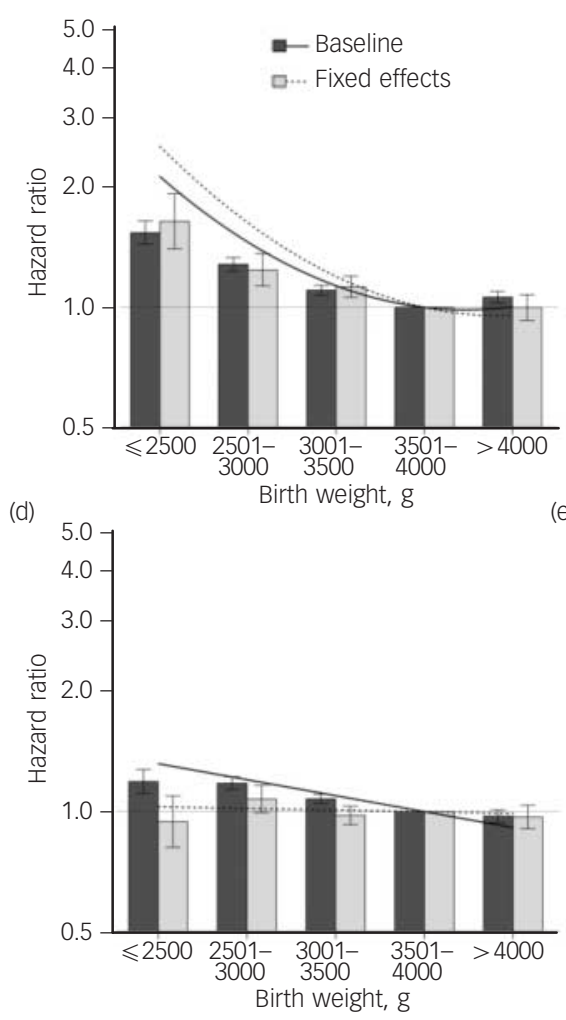
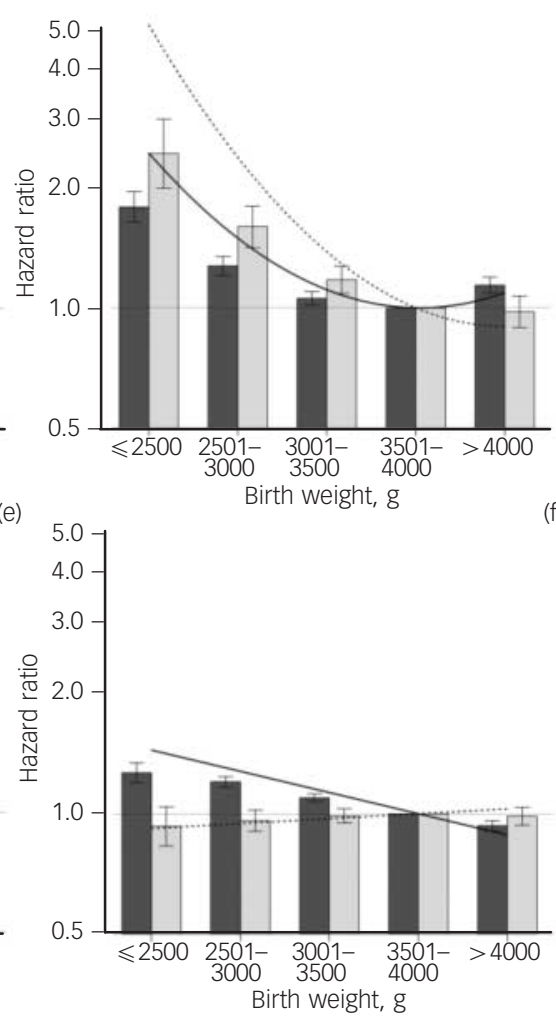

(c)
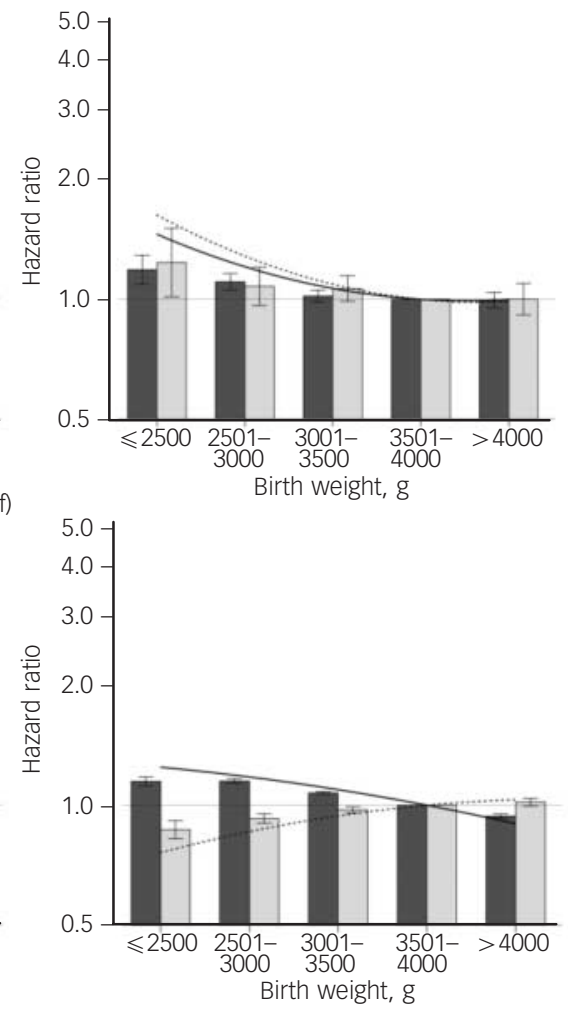

Fig. 1 Associations derived from continuous (line) and ordinal (bar with 95\% confidence interval) representation of birth weight when predicting psychiatric outcomes. (a) Attention-deficit hyperactivity disorder (ADHD), (b) autism spectrum disorder (ASD), (c) psychotic or bipolar disorder, (d) suicide attempt, (e) substance use problems, and
(f) criminality. Baseline, population-wide estimates are shown with the solid line and dark bars. Sibling-comparison, fixed-effects models are shown with dotted lines and light bars Reference group are those born in the $3501-4000 \mathrm{~g}$ birth weight category. The maintenance of association magnitude across population and sibling-comparison models, consistent with a causal inference can be noted when predicting ADD. ASD and psychotic or bipolar disorder only. A protective effect can be noted when predicting criminality in the decrease of association in the sibling-comparison model.

$\left(\mathrm{HR}_{\mathrm{BW}: \leqslant 2500 \mathrm{~g}}=1.15,95 \%\right.$ CI $\left.1.12-1.18\right)$, the direction of association switched in fixed-effects models. In the fixed-effects models, lower birth weight was slightly protective against criminality $\left(\mathrm{HR}_{\mathrm{BW}: \leqslant 2500 \mathrm{~g}}=0.87,95 \%\right.$ CI $\left.0.83-0.92\right)$.

As can be seen in Fig. 1, all psychiatric outcomes except suicide attempt and substance use problems were better explained by a quadratic representation of birth weight (see online Table DS3 for Akaike information criterion for linear and quadratic models). Adjusted models are not presented here or in Fig. 1 for ease of interpretation. Parameter estimates across all ordinal bins are presented in online Table DS4.

\section{Socioeconomic outcomes}

Figure 2 (a)-(c) present findings across ordinal and continuous birth weight representation for failing grades, education under 10 years and social welfare receipt, respectively. Population estimates suggested that lower birth weight was associated with increased risk for failing grades $\left(\mathrm{HR}_{\mathrm{BW}: \leqslant 2500 \mathrm{~g}}=1.66,95 \% \mathrm{CI}\right.$ $1.62-1.71)$ and education under 10 years $\left(\mathrm{HR}_{\mathrm{BW}: \leqslant 2500 \mathrm{~g}}=1.46\right.$, 95\% CI 1.42-1.49). These are presented in Fig. 2 with dark bars (ordinal) and a solid line (continuous). Fixed-effects models showed attenuated, although consistent, results for failing grades $\left(\mathrm{HR}_{\mathrm{BW}: \leqslant 2500 \mathrm{~g}}=1.07,95 \% \mathrm{CI} 1.01-1.13\right)$ and education under 10 years $\left(\mathrm{HR}_{\mathrm{BW}: \leqslant 2500 \mathrm{~g}}=1.18,95 \% \mathrm{CI} 1.12-1.24\right)$, as seen in the light bars (ordinal) and dotted line (continuous) in the figure. Thus, the results lend support to fetal growth being in the causal path towards failing grades and education under 10 years. A different pattern emerged for social welfare receipt, however
(Fig. 2(c)). Although the population estimate for social welfare receipt showed that lower birth weights are associated with increased social welfare receipt $\left(\mathrm{HR}_{\mathrm{BW}: \leqslant 2500 \mathrm{~g}}=1.52,95 \%\right.$ CI 1.49-1.55), the relation was completely attenuated in the fixed-effects model $\left(\mathrm{HR}_{\mathrm{BW}: \leqslant 2500 \mathrm{~g}}=1.00,95 \%\right.$ CI $\left.0.95-1.05\right)$.

\section{Sensitivity analyses}

First, to test whether results were biased by premature births, we limited the sample to full-term births only. Online Fig. DS1 shows that associations are comparable with those found in the main analyses, thus premature births were not driving the associations found. Second, we predicted two additional outcomes related to our main socioeconomic outcomes, low income and higher education. From these analyses we obtained converging evidence about the robust association between fetal growth and decreased odds of educational attainment, as well as the fully attenuated relationship between fetal growth and economic stability (online Fig. DS2). Third, we performed analyses to address some of the assumptions of the sibling-comparison design. To address concerns about the generalisability of findings from offspring with siblings to those without, we compared the population estimates in families with multiple children to those with only one child. Online Fig. DS3 shows that baseline population estimates were not different between offspring with one or more siblings as compared with only children. To address concerns about the generalisability of the findings from differentially exposed sibling to other populations, we conducted cousin-comparisons. Online Fig. DS4 presents the cousin-comparison results showing a commensurate 


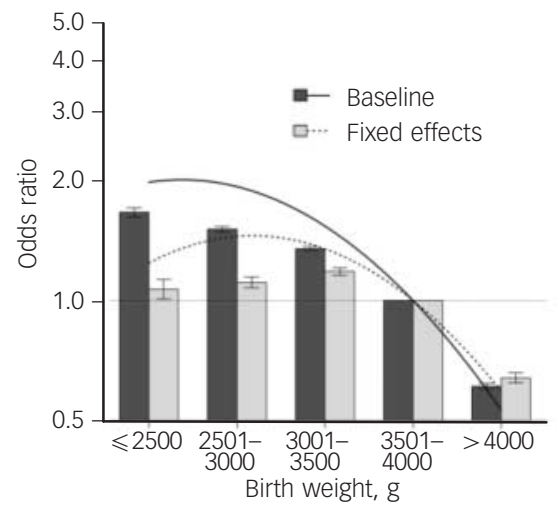

(b)

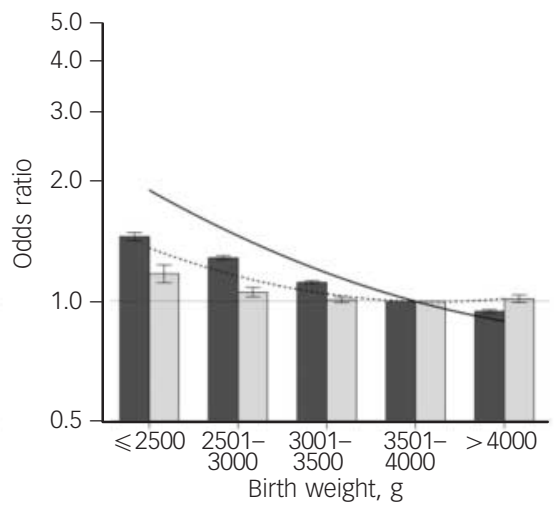

(c)

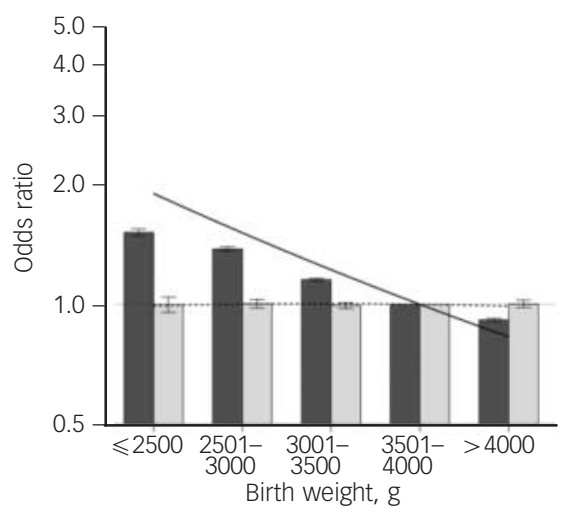

Fig. 2 Associations derived from continuous (line) and ordinal (bar with $95 \%$ confidence interval) representation of birth weight when predicting socioeconomic outcomes.

(a) Failing grades, (b) education under 10 years, and (c) social welfare receipt. Baseline, population-wide estimates are shown with the solid line and dark bars. Sibling-comparison, fixed-effects models are shown with dotted lines and light bars. Reference group are those born in the 3501-4000 g birth weight category. Although attenuated, the maintenance of association magnitude across population and sibling-comparison models, consistent with a causal inference, can be noted when predicting failing grades and education under 10 years only.

pattern of results to the main analyses. These results suggest that assumptions in sibling-comparison analyses (for example no carry-over effects) may not account for our conclusions.

\section{Discussion}

The current study examined the degree to which familial confounding, because of genetic and shared environmental factors, accounts for the associations between fetal growth, indicated by birth weight while controlling for gestational age, and psychiatric and socioeconomic problems. We used a large, well-validated, population-based data-set. The size and scope of the data-set provided us with the opportunity to examine rare and serious outcomes while studying the specificity of our findings across a broad range of outcomes. Further, the data structure allowed us to utilise quasi-experimental designs (for example sibling- and cousin-comparison) with precise measures of predictors and covariates. This is one of the first studies on fetal growth to have the power to examine associations using a sibling-comparison design, an important step towards supporting or refuting causal inferences.

Across outcomes, and in agreement with most previous research, ${ }^{2-8,26,39}$ the population estimates suggested that impaired fetal growth, as evidenced by lower birth weights, was associated with greater risk of each outcome. Results from sibling-comparison analyses showed that associations are consistent with causal inferences in an outcome-dependent manner. After fitting sibling-comparison fixed-effects models, the results support causal inferences between fetal growth and ASD, as well as ADHD. Despite some attenuation in fixed-effects models, the relation between fetal growth and psychotic or bipolar disorder, as well as failing grades and education less than 10 years, also supports a causal inference. Therefore, genetic and/or environmental factors specific to fetal development, as indexed by lower birth weight, influence the likelihood of these outcomes. In contrast, our results showed attenuation of the associations between fetal growth and suicide attempt, substance use problems and social welfare receipt, thus suggesting that these associations are primarily as a result of selection effects correlated with fetal growth. Additionally, sensitivity analyses provided evidence against alternative explanations for the findings.

\section{Associations consistent with a causal inference}

The associations between birth weight and ADHD and ASD were independent of shared familial confounds and statistical covariates, as the magnitudes of association remained significantly elevated in fixed-effects models. Although some previous research has reported null findings, ${ }^{1,5,18}$ the current results build on previous co-twin control ${ }^{2,4,10-12}$ and epidemiological study findings. ${ }^{3}$ The associations between birth weight and psychotic or bipolar disorder were also independent of shared familial confounds and statistical covariates, although the magnitudes of association were attenuated in fixed-effects models. Even more attenuated, although still present, were the associations between birth weight and educational attainment variables in the fixedeffects models. ${ }^{5-8,39}$ The interpretation of sensitivity analyses that examined the associations in full-term births only, in families with only one child and when comparing differentially exposed cousins (see online Fig. DS1, DS3, and DS4, respectively) did not differ from the main results. Therefore, overall, our findings lend greater support to the conclusion that fetal growth is along a causal pathway for these outcomes. Our findings also complement previous sibling-comparison research focusing on the long-term outcomes following early gestational age at birth. ${ }^{26}$

Comparing the associations across these outcomes, fetal growth appears to be more strongly related to early-onset neurodevelopmental disorders such as ADHD and ASD than for distal markers of neurodevelopmental problems, such as educational problems and later-onset disorders, such as psychotic or bipolar disorder. As evidence across studies converge on a consistent picture of the role of fetal growth on these outcomes, future research must examine possible mediating mechanisms. For example, previous research has shown white matter abnormalities because of brain injury associated with low birth weight. ${ }^{40}$ Other differences in brain development that correspond with neurodevelopmental problems, such as the amount of cortical surface area, brain volume and caudate volume, have also been noted even across variations within normal birth weight. ${ }^{41}$ Poor in utero nutrition may also be contributing to different fetal growth and altered brain development. ${ }^{42}$ ADHD and ASD have been shown to share common genetic aetiology, ${ }^{43}$ which will also have to be explored.

We also found that impaired fetal growth was associated with a decreased likelihood of criminality after fixed-effects modelling 
and across the sensitivity analyses. This result supports previous fetal growth $^{5}$ and gestational age research. ${ }^{26,44}$ Fetal-growthimpaired individuals may display personality characteristics linked with decreased risk-taking behaviours, receive increased parental monitoring and/or form fewer relationships with delinquent peers. Investigating violent $v$. non-violent crimes may elucidate the association further. ${ }^{45}$

\section{Associations fully attenuated}

Once the genetic and environmental factors that siblings share were controlled, the associations between fetal growth and substance use problems, suicide attempt and social welfare receipt were fully attenuated.

We also found converging evidence for social welfare receipt when predicting low income (see online Fig. DS2). The lack of association with substance use problems is in contrast to a previous co-twin control ${ }^{21}$ and an epidemiological ${ }^{5}$ study showing heavier infants were at increased risk for alcohol and drug use than lower birth weight infants, although measurement varies across studies.

\section{Strengths and limitations}

The sibling-comparison design allowed us to begin to address possible genetic confounding ${ }^{46}$ while also offering improved generalisability from a co-twin control approach. Fetal growth differences in twins may be aetiologically distinct from fetal grown differences between singletons, and twins have a greater risk for growth restriction in utero than singletons. ${ }^{27}$ Through the sensitivity analyses, we also explicitly tested some assumptions of the siblingcomparison design. ${ }^{24}$ Other sensitivity analyses included examining whether associations were driven by gestational age extremes, searching for converging evidence across related outcomes, and using continuously measured birth weight. Further, it should be noted that although our predictor was birth weight, we adjusted all associations for gestational age at birth. Therefore, we consider the predictor an index of fetal growth.

Despite these strengths, however, several limitations must be considered and addressed in future research. Sibling comparisons are not randomised controlled studies; therefore, the design cannot rule out all possible confounding factors and causation cannot be proven. Independent risk associated with fetal growth factors have been shown by comparing birth-weight-discordant monozygotic twins, ${ }^{2}$ which suggest such genetic factors do not explain the associations. Fixed-effects models also have lower statistical power than population-based estimates. ${ }^{47}$ Additional quasi-experimental research that relies on methods with different assumptions and limitations than the sibling-comparison approach is warranted. ${ }^{23}$ Replication in other countries, especially in countries differing in healthcare availability, is also needed.

\section{Implications}

Our findings contribute to the aetiological theory of neurodevelopmental disorders and socioeconomic outcomes, as causal inferences were divided by outcome type. The results suggest that efforts be made to reduce the incidence of low-birth-weight births. Results also call for public health initiatives providing services that target risks co-occurring with impaired fetal growth, as the associations between birth weight and substance use problems, suicide attempt, and social welfare receipt were as a result of selection factors that co-occur with birth weight. Further, our findings open an interesting line for future researchers to explore what factors associated with impaired fetal growth contribute to the decreased risk of criminality we identified in sibling-comparison analyses. Overall, the current study emphasises the importance of continued research on the role of fetal growth factors in offspring psychiatric and socioeconomic problems.

Quetzal A. Class, BS, Martin E. Rickert, PhD, Department of Psychological and Brain Sciences, Indiana University, Bloomington, Indiana; Henrik Larsson, PhD, Paul Lichtenstein, PhD, Department of Medical Epidemiology and Biostatistics, Karolinska Institutet, Stockholm, Sweden; Brian M. D'Onofrio, PhD, Department of Psychological and Brain Sciences, Indiana University, Bloomington, Indiana

Correspondence: Quetzal A. Class, Department of Psychological and Brain Sciences, Indiana University, 1101 East 10th St, Bloomington, IN 47405, USA. Email: qaclass@indiana.edu

First received 19 Dec 2013, final revision 1 Jun 2014, accepted 6 Jun 2014

\section{Funding}

This study was supported by grants from the National Institute of Mental Health (MH094011), National Institute of Child Health and Development (HD061817), the Swedish Council for Working Life and Social Research and the Swedish Research Council (Medicine).

\section{References}

1 Hack M, Youngstrom EA, Cartar L, Schluchter M, Taylor HG, Flannery D, et al. Behavioral outcomes and evidence of psychopathology among very low birth weight infants at age 20 years. Pediatrics 2004; 114: 932-40.

2 Losh M, Esserman D, Anckarsater H, Sullivan PF, Lichtenstein P. Lower birth weight indicates higher risk of autistic traits in discordant twin pairs. Psychol Med 2012; 42: 1091-102.

3 Hack M, Taylor HG, Schluchter M, Andreias L, Drotar D, Klein N. Behavioral outcomes of extremely low birth weight children at age 8 years. J Dev Behav Pediatr 2009; 30: 122-30.

4 Ficks CA, Lahey BB, Waldman ID. Does low birth weight share common genetic or environmental risk with childhood disruptive disorders? J Abnorm Psychol 2013; 122: 842-53.

5 Hack M, Flannery D, Schluchter M, Cartar L, Borawski E, Klein N. Outcomes in young adulthood for very-low-birth-weight infants. N Engl J Med 2002; 346: 149-57.

6 Aarnoudse-Moens CSH, Weisglas-Kuperus N, van Goudoever B, Oosterlaan J. Meta-analysis of neurobehavioral outcomes in very preterm and/or very low birth weight children. Pediatrics 2009; 124: 717-28.

7 Lawlor DA, Bor W, O'Callaghan MJ, Williams GM, Najman JM. Intrauterine growth and intelligence within sibling pairs: findings from Mater-University study of pregnancy and its outcomes. J Epidemiol Community Health 2005; 59: $279-82$.

8 Matte TD, Bresnahan M, Begg MD, Susser ES. Influence of variation in birth weight within normal range and within sibships on IQ at age 7 years: cohort study. BMJ 2001; 323: 310-4.

9 Barker DJP. Mothers, Babies and Health in Later Life (2nd edn). Churchill Livingstone, 1998.

10 Groen-Blokhuis MM, Middeldorp CM, van Beijsterveldt CEM, Boomsma DI. Evidence for a causal association of low birth weight and attention problems. J Am Acad Child Adolesc Psychiatry 2011; 50: 1247-54.

11 Hultman CM, Torrang A, Tuvblad C, Cnattingius S, Larsson J, Lichtenstein P. Birth weight and attention-deficit/hyperactivity symptoms in childhood and early adolescence: a prospective Swedish Twin study. J Am Acad Child Adolesc Psychiatry 2007; 46: 370-7.

12 Lehn H, Derks EM, Hudziak JJ, van Beijsterveldt TC, Boomsma DI. Attention problems and attention-deficit/hyperactivity disorder in discordant and concordant monozygotic twins: evidence of environmental mediators. J Am Acad Child Adolesc Psychiatry 2007; 46: 83-91.

13 Thapar A, Rutter M. Do prenatal risk factors cause psychiatric disorder? Be wary of causal claims. Br J Psychiatry 2009; 195: 100-1.

14 Smith GD. Assessing inrauterine influences on offspring health outcomes: can epidemiological studies yield robust findings? Basic Clinic Pharmacol Toxicol 2008; 102: 245-56.

15 Weightman AL, Morgan HE, Shepherd MA, Kitcher H, Roberts C, Dunstan FD. Social inequality and infant health in the UK: systematic reviews and meta-analyses. BMJ Open 2012; 2: e000964.

16 Lunde A, Melve KK, Gjessing HK, Skjaerven R, Irgens LM. Genetic and environmental influences on birth weight, birth length, head circumference, and gestational age by use of population-based parent-offspring data. Am J Epidemiol 2007; 165: 734-41. 
17 Saigal S, Stoskopf B, Streiner D, Boyle M, Pinelli J, Paneth N, et al Transition of extremely low-birth-weight infants from adolescence to young adulthood, comparison with normal birth-weight controls. JAMA 2006; 295: 667-75.

18 Ronald A, Happe F, Dworzynski K, Bolton P, Plomin R. Exploring the relation between prenatal and neonatal complications and later autistic-like features in a representative community sample of twins. Child Dev 2010; 81: 166-82.

19 Wojcik W, Lee W, Colman I, Hardy R, Hotopf M. Foetal origins of depression? A systematic review and meta-analysis of low birth weight and later depression. Psychol Med 2013; 43: 1-12.

20 Lawlor DA, Clark H, Smith GD, Leon DA. Intrauterine growth and intelligence within sibling pairs: findings from the Aberdeen children of the 1950s cohort. Pediatrics 2006; 117: e894-902.

21 Foley DL, Neale MC, Kendler KS. Does intra-uterine growth discordance predict differential risk for adult psychiatric disorder in a population-based sample of monozygotic twins? Psychiatr Genet 2000; 10: 1-8.

22 Kramer MS. Invited Commentary: association between restricted fetal growth and adult chronic disease: is it causal? Is it important? Am J Epidemiol 2000; 152: 605-8.

23 Rutter M. Proceeding from observed correlation to causal inference: the use of natural experiments. Perspect Psychol Sci 2007; 2: 377-95.

24 Lahey BB, D'Onofrio BM. All in the family: comparing siblings to test causal hypotheses regarding environmental influences on behavior. Curr Dir Psychol Sci 2010; 19: 319-23.

25 D'Onofrio BM, Lahey B, Turkheimer E, Lichtenstein P. Critical need for family-based, quasi-experimental designs in integrating genetic and social science research. Am J Public Health 2013; 103: S46-55.

26 D'Onofrio BM, Class QA, Rickert ME, Larsson H, Langstrom N, Lichtenstein P. Preterm birth and mortality and morbidity: a quasi-experimental study. JAMA Psychiatry 2013; 70: 1231-40.

27 Loos RJ, Derom R, Vlietinck R. Determinants of birthweight and intrauterine growth in liveborn twins. Paediatr Perinat Epidemiol 2005; 19: 15-22.

28 Indring S, Rai D, Dal H, Dalman C, Sturm H, Zander E, et al. Autism spectrum disorders in the Stockholm Youth Cohort: design, prevalence and validity. PLOS One 2012; 7: e41280.

29 Lichtenstein P, Yip BH, Bjork C, Pawitan Y, Cannon T, Sullivan PF, et al. Common genetic determinants of schizophrenia and bipolar disorder in Swedish families: a population-based study. Lancet 2009; 373: 234-9.

30 D'Onofrio BM, Rickert ME, Langstrom N, Donahue KL, Coyne CA, Larsson H, et al. Familial confounding of the association between maternal smoking during pregnancy and offspring substance use and problems: converging evidence across samples and measures. Arch Gen Psychiatry 2012; 69: $1140-50$.

31 Tidemalm D, Langstrom N, Lichtenstein P, Runeson B. Risk of suicide after suicide attempt according to coexisting psychiatric disorder: Swedish cohort study with long term follow-up. BMJ 2008; 337: 1-6.
32 D'Onofrio BM, Singh AL, lliadou AN, Lambe M, Hultman CM, Grann M, et al. Familial confounding of the association between maternal smoking during pregnancy and offspring criminality: a population-based study in Sweden. Arch Gen Psychiatry 2010; 67: 529-38.

33 Larsson H, Chang Z, D'Onofrio BM, Lichtenstein P. The heritability of clinically diagnosed attention-deficit/hyperactivity disorder across the life span. Psychol Med 2013; Oct 10: 1-7 (epub ahead of print).

34 World Health Organization. International Statistical Classification of Diseases and Related Health Problems (ICD-9). WHO, 1978.

35 World Health Organization. The ICD-10 Classification of Mental and Behavioural Disorders: Clinical Descriptions and Diagnostic Guidelines. WHO, 1992

36 World Health Organization. International Statistical Classification of Diseases and Related Health Problems (ICD-8). WHO, 1967.

37 D'Onofrio BM, Singh A, lliadou AN, Lambe M, Hultman CM, Neiderhiser JM et al. A quasi-experimental study of maternal smoking during pregnancy and offspring academic achievement. Child Dev 2010; 81: 80-100.

38 Statistics Sweden. Educational Attainment of the Population. Statistics Sweden (www.scb.se/pages/subjectarea___3930.aspx, accessed 14 August 2013).

39 Newcombe R, Milne BJ, Caspi A, Poulton R, Moffitt TE. Birthweight predicts IQ: fact or artefact? Twin Res Hum Genet 2007; 10: 581-6.

40 Skranes J, Evensen KI, Lohaugen GC, Martinussen M, Kulseng M, Myhr G, et al. Abnormal cerebral MRI findings and neuroimpairments in very low birth weight (VLBW) adolescents. Eur J Paediatr Neurol 2009; 12: 273-83.

41 Walhovd KB, Fjell AM, Brown TT, Kuperman JM, Chung Y, Hagler Jr DJ, et al. Long-term influence of normal variation in neonatal characteristics on human brain development. Proc Natl Acad Sci USA 2012; 109: 20089-94.

42 de Bie HM, Oostrom KJ, Delemarre-van de Waal HA. Brain development, intelligence and cognitive outcome in children born small for gestational age. Horm Res Paediatr 2010; 73: 6-14.

43 Pettersson E, Anckarsater H, Gillberg C, Lichtenstein P. Differen neurodevelopmental symptoms have a common genetic etiology. J Child Psychol Psychiatry 2013; 54: 1356-65.

44 Hack M. Adult outcomes of preterm children. J Dev Behav Pediatr 2009; 30 460-70.

45 Kuja-Halkola R, Pawitan Y, D'Onofrio BM, Langstrom N, Lichtenstein P. Advancing paternal age and offspring violent offending: a sibling-comparison study. Dev Psychopathol 2012; 24: 739-53.

46 Bennedsen BE, Mortensen PB, Olesen AV, Henriksen TB. Preterm birth and intra-uterine growth retardation among children of women with schizophrenia. Br J Psychiatry 1999; 175: 239-45.

47 Allison PD. Fixed Effects Regression Models. Sage, 2009. 